Revista Venezolana de Gerencia (RVG)

Año 13. $N^{\circ} 44,2008,655$ - 668

Universidad del Zulia (LUZ) • ISSN 1315-9984

\title{
Internet y televisión por cable en Venezuela: Una mirada estratégica*
}

\author{
Queipo, Beatriz ** \\ Artigas, Wileidys *** \\ Useche, María **** \\ Romero, Mildred *****
}

\section{Resumen}

El direccionamiento estratégico en la organización del trabajo de las empresas que operan en el sector de las telecomunicaciones en Venezuela ha sufrido modificaciones, debido a que el mismo se encuentra en un ambiente dinámico, que implica una continua mejora tecnológica; y por tanto, sus procesos se ven afectados y reformados en base a las mismas. En este sentido, los segmentos de Internet y televisión por cable, han estado adaptándose al crecimiento progresivo, estableciendo estrategias operativas que determinan su permanencia y competitividad en el mercado. Ello motivó a clasificar las estrategias implementadas en la organización del trabajo de las empresas de dichos sectores. El tipo de investigación fue descriptiva y diseño de campo. Teóricamente el estudio se sustentó en la escuela de posicionamiento bajo la perspectiva de Porter (2000), así como, Thompson y Strickland (2004). Los datos fueron recogidos mediante entrevistas semiestructuradas aplicadas directa-

Recibido: 27-11-07. Aceptado: 28-03-08

* Resultados finales del proyecto de Investigación titulado: Estrategias de Modernización en la Organización del Trabajo en Empresas de Telecomunicaciones en el Estado Zulia. Segunda Parte. Financiado por el Consejo de Desarrollo Científico y Humanístico de la Universidad del Zulia, No. CH-1129-2006. Maracaibo, Venezuela.

** Investigadora. Centro de Estudios de la Empresa. Facultad de Ciencias Económicas y Sociales. Universidad del Zulia. Licenciada en Administración. Estudiante de la Maestría en Gerencia de Proyectos de Investigación y Desarrollo. E-mail: vicqueipo@yahoo.com.

*** Coinvestigadora. Centro de Estudios de la Empresa. Facultad de Ciencias Económicas y Sociales. Universidad del Zulia. Docente. Universidad Rafael Belloso Chacín. Licenciada en Administración y Magíster en Gerencia de Empresas, mención Gerencia de Operaciones.

E-mail: wileidys@hotmail.com.

**** Investigadora-Docente. Centro de Estudios de la Empresa. Facultad de Ciencias Económicas y Sociales. Universidad del Zulia. Economista y Magíster en Gerencia de Empresas, Mención Mercadeo. Estudiante del Doctorado de Ciencias Económicas.

E-mail: mariauseche@yahoo.es.

***** Coinvestigadora-Docente. Centro de Estudios de la Empresa. Facultad de Ciencias Económicas y Sociales. Universidad del Zulia. Licenciada en Administración y Magíster en Gerencia de Empresas, Mención Industrial. E-mail:mildredgenove@yahoo.com. 
Internet y televisión por cable en Venezuela: Una mirada estratégica

Queipo, Beatriz; Artigas, Wileidys; Useche, María y Romero, Mildred

mente en las empresas, a saber: CANTV, Net Uno, Intercable, Procedatos y Movistar. Se concluyó, según la determinación de grupos estratégicos, que en su mayoría la orientación estratégica de las empresas de Internet y televisión por cable es hacia el posicionamiento por costos, buscando la eficiencia operativa y una mayor rentabilidad en el mercado por medio del ofrecimiento de precios más bajos, como consecuencia de la disminución de los costos de operación.

Palabras clave: Organización del trabajo, estrategias, costos, posicionamiento, telecomunicaciones.

\title{
Internet and Cable TV in Venezuela: A Strategic Look
}

\begin{abstract}
Strategic direction in the organization of work for companies that operate in the telecommunications sector in Venezuela has suffered modifications, because the sector is immersed in a dynamic environment, which implies continuous technological improvement; therefore, its processes are affected and reformed on the basis of the same. In this respect, the Internet and cable television segment has been adapting to the progressive growth, establishing operative strategies that determine its permanency and competitiveness in the market. This motivated a classification of the strategies implemented in organizing the work of the companies in the above-mentioned sectors. The type of investigation was descriptive with a field design. Theoretically, the study was based on the school of positioning from Porter's perspective (2000), as well as that of Thompson and Strickland (2004). Information was gathered by means of semi-structured interviews applied directly in the companies, namely: CANTV, Net Uno, Intercable, Procedatos and Movistar. It was concluded that, according to the determination of strategic groups, in the main, the principal strategic orientation of Internet and cable TV companies is toward positioning by costs, seeking operative efficiency and greater profitability in the market by offering the lowest prices, as a consequence of decreasing operational costs.
\end{abstract}

Key words: Labor organization, strategies, costs, positioning, telecommunications.

\section{Introducción}

Las telecomunicaciones en el ámbito internacional han tomado un gran auge en los últimos años, como consecuencia de la velocidad de procesamiento de información requerida en los diferentes sectores de la economía, sobre todo, en el caso de internet, ya que conforma herramientas con un elevado valor de mercado, por su intervención real en los mecanismos de distribución y asignación de recursos, materias primas, comercialización, entre otros.
En el caso de Venezuela, la apertura del sector de telecomunicaciones en el año 2000 estimuló la inversión en tecnología, y como consecuencia de ello las empresas que funcionan en los rubros de Internet y televisión por cable en el país han venido modificando paulatinamente la organización del trabajo, con la finalidad de agilizar los procesos operativos y reducir los costos para responder competitiva y oportunamente a los cambios del mercado y a la competencia nacional. 
En este sentido, la dirección estratégica funciona como el elemento mediador para el uso adecuado de los avances del mercado y el logro de la adaptación al mismo; por lo cual, al estudiar las estrategias utilizadas se puede observar el funcionamiento de los elementos estratégicos dentro de dichas organizaciones; y directamente, en la organización del trabajo.

En efecto, las estrategias son cursos de acción que establece la organización en sus distintos niveles (corporativo, de negocios, funcionales u operativos), pero referidas a lineamientos específicos que determinaran la eficiencia o la calidad como sus fines principales (liderazgo en costes, diferenciación o nichos de mercado). Por lo cual, en base a esta perspectiva, se procederá a realizar la clasificación de las estrategias implementadas en las empresas de Internet y suscripción por cable en Venezuela.

A través de este trabajo fue posible conocer las prácticas y cursos de acción aplicados por las empresas; así como, considerar la interrelación entre la variable y los elementos estudiados y la correspondencia de la empíria con los enfoques teóricos de la administración científica, especialmente en cuanto a administración estratégica. Para lo cual, el trabajo fue desarrollado mediante un tipo de investigación descriptivo, presentando las estrategias tal cual como fueron recolectadas en el mercado.

El diseño de investigación fue de campo, recolectando los datos de la realidad y presentándolos tal cual como se observaron. Como técnica se llevo a cabo la entrevista, mediante el uso de un cuestionario semiestructurado aplicado a los gerentes y cargos medios de las empre- sas en cuestión, dichos cargos relacionados directamente con las áreas de personal, operaciones o mercadeo, específicamente los encargados del establecimiento de las estrategias en las referidas áreas, contrastando así, las diferentes opiniones y puntos de vistas del personal. Es importante, dejar claro que las entrevistas se identifican con los nombres de las empresas a fin de mantener la confidencialidad de las personas entrevistadas en las empresas, en relación a la información suministrada.

Con base a lo anterior, se describen las estrategias detectadas en la recopilación de la información, para luego identificar la tipología a la que responden, a través del enfoque de la escuela de posicionamiento de Porter (2002), así como, Thompson y Strickland (2004), por lo cual, se puede decir, que el trabajo pretende contrastar la teoría de dichos autores con la empíria; presentando las estrategias conjuntamente con la teoría utilizada.

\section{Estrategias implementadas por las empresas de Internet y Televisión por cable en Venezuela.}

El presente apartado, se inicia describiendo las estrategias empleadas por los operadores, basados en la concepción de Porter (2002), para establecer sí las mismas se encuentran orientadas estratégicamente al liderazgo en costos, la diferenciación o la concentración; así como, los niveles sobre los cuales fueron establecidas, a saber: corporativo, de negocio funcional y operativas (Thompson y Strickland, 2004).

Es importante destacar, que los nombres asignados a las estrategias han 
Internet y televisión por cable en Venezuela: Una mirada estratégica Queipo, Beatriz; Artigas, Wileidys; Useche, María y Romero, Mildred

sido producto de un consenso con el grupo de investigación en función de los elementos agrupados dentro de cada una de ellas, es decir, no responden a nomenclaturas obtenidas de categorías teóricas. La agrupación y descripción de las estrategias se llevaron a cabo tomando en cuenta las que fueron implementaron ambas empresas, las cuales se denominan "grupos estratégicos" según Porter (2000). trabajo

a)Estrategia especialización del

De acuerdo a la información recolectada el tipo de especialización encontrado fue funcional, por medio del cual se asignan tareas de forma individual, en base a una función especifica (producción, ventas, finanzas o mercadeo) y dependiendo de la posición o cargo. El agrupamiento funcional que aplican estas empresas permite que los empleados desarrollen un conocimiento específico, habilidades y destrezas, pero sólo dentro de la función desarrollada.

La acción de agrupar labores por especialidad funcional se suele designar forma de organización unitaria o forma $U$, porque coloca cada función primaria en una unidad de negocio principal. Las labores individuales están caracterizadas por la asignación de tareas; por ejemplo, todas las labores de ventas de la organización se agrupan para formar un departamento de ventas y todas las labores de servicio se agrupan para formar el departamento de servicio, éstos departamentos se encargan de administrar sus funciones particulares para aplicarlas a las línea entera de productos de la empresa (Brickley et al., 2005: 118).

Con esta estrategia, las operadoras que prestan el servicio de Internet y televi- sión por cable buscan fomentar la coordinación de actividades efectiva en las áreas operativas. En Procedatos (2007); Movistar (2007); CANTV (2007) y Net Uno (2007) el jefe de departamento asigna empleados a tareas específicas con base a su actual carga de trabajo y experiencia; logrando con esto fomentar la experiencia operativa, definir una ruta de ascenso hacia puestos superiores para los empleados y permitiendo utilizar las habilidades de la persona. En Procedatos, CANTV, Net Uno y Movistar se pudo observar que a pesar de la asignación de tareas en forma individual no excluye la posibilidad de otorgar apoyo entre cargos, lo cual hace que el supervisor pueda asignar de un momento a otros empleados en función de las necesidades.

Para Koontz y Weihrich (1994: 268) el agrupamiento de actividades de acuerdo con las funciones de una empresa abarca todo lo que las empresas hacen normalmente. En éstas empresas las tareas se encuentran enumeradas y especificadas en los manuales de descripción de cargos. Esta agrupación trae desventajas para las empresas, como problemas significativos de coordinación entre departamentos ocasionando largas demoras en el servicio de atención al cliente. Dando como resultado, que en vista a la multiplicidad de actividades a desarrollar, los empleados se concentren en su meta individual en detrimento de la meta global.

Según entrevistas realizadas a CANTV (2007), Movistar (2007) y Net Uno (2007) se pudo constatar que los empleados están concentrados a sus cargos y departamentos; por ejemplo, los empleados que se encuentran en mercadeo no muestran interés o en muchos casos 
desconocen la importancia de una actividad que requiere el departamento de ventas y no le brinda su apoyo para su realización, siendo esto una desventaja donde cada trabajador o área funcional está concentrado en su tarea, y lo importante es alcanzar o cumplir con sus obligaciones, enfocándose en lo particular y olvidándose de los resultados generales, nadie se hace responsable de los resultados de la institución como un todo, y por ello, la dificultad de coordinar las diferentes "islas" en que se convierten cada unidad funcional.

En función de la clasificación, en cuanto a la especialización del trabajo, las empresas de televisión por cable e Internet en Venezuela asignan tareas de forma individual según el cargo ocupado (CANTV, 2007; Intercable, 2007; Net Uno, 2007; Movistar, 2007), basándose en una división del trabajo de tipo funcional, concentrando las labores del personal y logrando la especialización del trabajo, con lo cual, se observó, que esta división vuelve al individuo muy centrado en su actividad o función, dejando de lado las actividades que se desarrollan a su alrededor, es decir, individualizando el trabajo, a excepción de ciertos casos (Procedatos, 2007) en los que al trabajar por proyectos se fomenta el desarrollo del trabajo grupal.

Esta estrategia se inserta dentro de la clasificación de diferenciación de las estrategias de Porter (2000), la misma garantiza que el servicio llegue de forma eficiente al cliente mediante personal que cuenta con una especificación de sus funciones, pero al mismo tiempo debe tenerse cuidado en función de que al llegar a la superespecialización se puede incurrir en la desvinculación de los individuos del resto de los cargos, que en conjunto representan los procesos completados y el logro de los objetivos de la organización.

Tomando en cuenta los niveles en los cuales se establece la estrategia, se puede decir que la misma se encuentra entre las estrategias de negocio de Thompson y Strickland (2004) debido a que se establece para definir el negocio, ya que la organización como negocio tiene establecidos manuales y funciones específicas en relación de las áreas que se manejan, por lo cual, ya la estructura, cargos y funciones se encuentran preestablecidos desde las sedes principales.

b)Estrategia indicadores de medición.

Para medir el desempeño de una empresa o unidad ya sea en calidad, productividad, costo, seguridad, entre otros, se necesita tener herramientas de medición. Entre ellas, el indicador de gestión es la expresión cuantitativa del comportamiento o desempeño de una empresa o departamento, cuya magnitud, al ser comparada con algún nivel de referencia, podrá estar señalando una desviación sobre la cual se tomarán acciones correctivas o preventivas según el caso (Corporación Andina de Fomento, 1991:34).

Un sistema de medición funciona como un panel de control para que la organización o cada departamento pueda según Chiavenato (2006) evaluar el desempeño e indicar las acciones correctivas necesarias, apoyar la mejora del desempeño, mantener la convergencia de propósito y la herencia de esfuerzo en la organización de la integración de estrategia, garantizando acciones futuras que posibiliten alcanzar mejores resultados. 
Internet y televisión por cable en Venezuela: Una mirada estratégica Queipo, Beatriz; Artigas, Wileidys; Useche, María y Romero, Mildred

Las empresas CANTV (2007); Net Uno (2007); Movistar (2007); Procedatos (2007) e Intercable (2007) emplean indicadores para medir el desempeño de los trabajadores y el servicio, entre los indicadores que aplican se encuentran:

- Ejecución del plan.

- Tiempo de atención al cliente.

- Disponibilidad del servicio.

- Medición de clima organizacional. Las empresas de televisión por cable e Internet manejan los indicadores de desempeño en relación a la evaluación de desempeño, que es obligatoria a toda la organización, evaluando en los empleados elementos como eficiencia, eficacia y productividad, ello para medir sí los empleados cumplieron las metas y en el tiempo establecido. En Procedatos (2007) y Movistar (2007) se consideran como referencia, para la definición de los porcentajes de aumento de salario que se dan de forma anual, del mismo modo, tanto Procedatos (2007) como Net Uno (2007) señalan que esto no excluye que algunos supervisores pueden tener su propia evaluación de desempeño y que ésta sea aplicada con mayor frecuencia que la obligatoria.

La estrategia de indicadores de medición permite asegurar el logro de metas y objetivos, mediante la medición continua de los estándares, como el caso de eficiencia, productividad, entre otros. Los indicadores de medición son establecidos en función de observar el desempeño de cada unidad para asegurar el logro de los objetivos organizacionales (CANTV, 2007; Intercable, 2007; Net Uno, 2007 y Procedatos, 2007), sin dejar de lado, que en algunos casos se llevan a cabo en función de lineamientos internacionales (Movistar, 2007).
Esta estrategia se inserta dentro de las estrategias genéricas de liderazgo en costos, debido a que en la búsqueda del cumplimiento de las metas y al asegurar el logro de los objetivos y estándares preestablecidos se logra la reducción de los costos asociados a los retrabajos y retrasos en el cumplimiento de dichos objetivos. Por otro lado, no debe olvidarse que mediante el establecimiento de indicadores en función de la evaluación del desempeño, se fomenta la competitividad interna para el logro de un mejor servicio que resultará en beneficios al cliente. Sin dejar de lado que mediante la obtención de los índices puede fomentarse el mejoramiento de los procesos sobre la marcha (Intercable, 2007).

Los indicadores son establecidos desde los altos niveles organizacionales (CANTV, 2007; Intercable, 2007; Net Uno, 2007; Movistar, 2007 y Procedatos, 2007), ubicándose dentro de las estrategias corporativas de Thompson y Strickland (2004), debido a que los indicadores de desempeño generalmente se ubican dentro de los lineamientos de las organizaciones internacionales y son traspoladas a las unidades de negocios para asegurar la eficiencia organizacional.

c)Estrategia rotación interna del personal

Mediante la rotación interna del personal se busca lograr la incursión del personal en diversas áreas o secciones del departamento o unidad del cual formaban parte, dicha incursión se denomina rotación o transferencia interna de personal, permitiendo contar con trabajadores y equipos de trabajo capaces de cumplir con sus funciones y tareas direc- 
tas; para que el proceso de trabajo continúe ante la falta de alguno de los trabajadores.

Estos elementos se solapan con los postulados de la teoría neoclásica, relacionados con la especialización, los cuales parten según Melinkoff (1990) de la idea de que los miembros de un grupo organizado podrán tener alcances y contenidos diversos, ya que han sido reformados por los adelantos técnicos de la organización y de la dirección, así como el desarrollo social y tecnológico que han hecho más flexible y productiva la especialización, acciones que se logran aprovechando las ventajas del asesoramiento y de la organización funcional.

Lo anterior permite que cada miembro de la organización pueda realizar más de una función, al contar con trabajadores que se desempeñaban como analistas y administradores integrales. Esto incrementó las habilidades y destrezas del personal, para ser más productivos y manejar correctamente las actividades y tareas de la unidad o departamento al cual pertenecían.

La rotación en las empresas de suscripción por cable e Internet, se lleva a cabo en función de las necesidades de sustitución o suplencia del personal y para ampliar conocimientos las mismas generalmente no vienen acompañadas de reclasificación ni de cambios en los ingresos, a menos que el cargo que se esté supliendo incluya ingresos mayores. También se evidenció que esta rotación se realiza por áreas, sobretodo en atención al cliente; con el fin de evitar vicios entre los trabajadores y la preferencia con los clientes.
Mediante esta estrategia se pretende que las personas tengan conocimiento acerca de los diferentes puestos del área donde se desenvuelven en función de cubrir cualquier eventualidad que se presente en la misma, como el caso de falta de personal, ausencias, vacaciones, despidos, entre otros. La rotación se realiza en función de asegurar la continuidad de los procesos (CANTV, 2007; Intercable, 2007; Net Uno, 2007; Movistar, 2007 y Procedatos, 2007), para alguno de los casos, la rotación se realizaba de forma obligatoria en el área de ventas y atención al cliente, con cronograma de actividades (Movistar, 2007).

La estrategia se inserta dentro de la clasificación de liderazgo en costos establecida por Porter (2000), ya que mediante el uso de personal en las diversas funciones de las áreas, sobretodo en cuanto a ventas y atención al cliente, se asegura el funcionamiento y eficiencia de las mismas. La estrategia de rotación en algún momento pudo haber sido una estrategia de diferenciación, pero en función de que las empresas fueron imitando las acciones en la búsqueda de la competitividad, se convirtió en una estrategia de liderazgo en costos al ir modificando la forma de aplicación en la búsqueda de la eficiencia.

En relación al nivel en el cual se desarrolla la estrategia, ésta es establecida operativamente (Thompson y Strickland, 2004), en referencia a actividades o tareas específicas dentro de las empresas, por lo cual, cada sucursal establece operativamente sus rotaciones en función de sus necesidades. 
Internet y televisión por cable en Venezuela: Una mirada estratégica Queipo, Beatriz; Artigas, Wileidys; Useche, María y Romero, Mildred

\section{d)Estrategia automatización de los procesos.}

La automatización de procesos, consiste en usar las mejoras en cuanto a tecnología que constantemente se dan en el sector, para automatizar los procesos de las empresas, en función de agilizar y mejorar constantemente dichos procesos. Como señala Gual (2001) el cambio tecnológico ha modificado la cadena de valor de las empresas de telecomunicaciones e Internet.

Con los drásticos cambios del entorno de los negocios que se han derivado del rápido cambio tecnológico y de una competencia global más intensa, todas las empresas enfrentan nuevos desafíos (Brickley y col., 2005:118). Desafíos que hacen que las empresas prestadoras del servicio de Internet apliquen la estrategia innovativa imitativa que según Molina y Conca (2000: 52) está relacionada con la compra de patentes y/o licencias, que permiten emplear las innovaciones desarrolladas por otras empresas.

Las innovaciones técnicas adquiridas por las empresas CANTV (2007); Net Uno (2007); Procedatos (2007); Intercable (2007) y Movistar (2007) han permitido automatizar sus procesos administrativos y operativos; como las solicitudes de servicio, nómina de personal, facturación, entre otros; para agilizar los procesos y minimizar los tiempos de autorización y de respuesta al mercado, producto de una mayor descentralización de actividades operativas rutinarias en la región.

Según las empresas, con esta estrategia se han logrado mejorar la reducción de tiempo de espera, agilizando los procesos de comunicación en las empre- sas y prestar servicios a los clientes con calidad. Esto producto de la incorporación de tecnologías de avance como son las herramientas de atención al cliente, como el caso de CVSC (Centro Virtual de Servicio al Cliente) y TBilling (facturación de cuentas de Internet), sistemas utilizado por Movistar (2007) en el cual se maneja la base de datos de los clientes y la facturación, respectivamente.

Por la tanto "la estrategia imitativa pueden implicar una actitud activa de la empresa, puesto que puede ser utilizada para adquirir un Know How (saber hacer), que en un mediano o largo plazo permita poner en práctica estrategias defensivas u ofensivas" (Molina y Conca, 2000: 52).

Por otro lado, la aplicación de las tecnologías en los procesos administrativos y operativos en estas empresas según Fernández y col. (2003) favorece la introducción de nuevos productos en el mercado, la prestación de productos personalizados y acomodados a los deseos de los clientes a unos costos accesibles con una segmentación más afinada, mayor precisión y capacidad de repetición de los procesos, lo que reduce la variabilidad del mismo y favorece una mayor calidad del servicio.

Al respecto, las empresas estudiadas automatizaron el funcionamiento de sus procesos, donde la ejecución del trabajo era menos compleja y se dotó al personal de herramientas tecnológicas adquiridas, reduciendo el trabajo realizado de forma manual, lo cual trajo como consecuencia la reducción de los costos asociados con el tiempo de respuestas de cada región ante el cliente, así como, los tiempos de autorización y solución de problemas. 
Se puede decir entonces, que la tecnología es determinante para la estabilidad y el éxito de las empresas "ya que condiciona la calidad y coste de sus productos y determina su competitividad (presente y futura), las cuotas del mercado y los resultados financieros" (Escorsa y Vall, 2003:61).

En líneas generales, las empresas que prestan el servicio de Internet y televisión por cable poseen sistemas y aplicaciones adaptados a sus necesidades o tamaño de la empresa; así, empresas con mayor capacidad de inversión poseen Sistemas, Aplicaciones y Procesos (SAP) y las otras poseen aplicaciones menos amplias pero con la misma finalidad: tener una base de datos del cliente, que permita el manejo del mismo. Es importante resaltar que ninguna posee un sistema o proceso automatizado que los diferencie, porque cuando una empresa introduce alguno como ventaja competitiva, la competencia inmediatamente se iguala.

Por lo tanto, en estos tiempos de fuerte competencia de precios para las operadoras resulta difícil diferenciar sus servicios de los competidores. Los clientes pueden percibir los servicios de diferentes proveedores tan similares que se preocupan menos por el proveedor que por el precio (Kotler et al., 2004).

Por lo que, según el mismo autor una de las mejores formas de diferenciación estratégica para la empresa es brindar permanentemente más calidad que sus competidores, como la tendencia de la calidad dirigida hacia el cliente. Aunado a esto, las operadoras han adquirido tecnologías para mejorar los procesos y servicios que les permita trasmitir al mismo tiempo datos, voz y videos a mayor velo- cidad con la finalidad de ofrecer superiores y nuevos servicios para satisfacer al cliente. Ello también, conducido a la mejora de los sistemas de información de manera de poder optimizar los servicios de cobranza, de compra, de reclamo con la finalidad de corregir los tiempos de respuestas.

Por otro lado, para Zeithaml y Bitner (2000) como los servicios se producen y se consumen en forma paralela y con frecuencia envuelven interacción entre empleados y clientes, también es primordial que el proceso de desarrollo de los servicios involucre tanto a los empleados como a los clientes.

Ante esto, según CANTV (2007) y Procedatos (2007) para el desarrollo de sus servicios al cliente, no sólo toman en cuentan la información que éste pueda brindar sobre sus necesidades, a través del sistema de atención al cliente donde el usuario puede realizar reclamos o sugerencias, y también consideran las opiniones de su trabajadores, dado que se encuentran en contacto con el cliente; y por lo tanto, pueden identificar los aspectos de organización que precisan ser abordados.

Además, se han introducido sistemas que han agilizado los procesos de atención al cliente, como el caso de SAP (CANTV, 2007; Movistar, 2007 y Procedatos, 2007). La utilización de la tecnología de avanzada, de forma constante para la prestación de los servicios de Internet y televisión por cable de forma interna en las organizaciones garantiza de cierta forma la agilización de los procesos internos.

Ésta estrategia se inserta dentro de la clasificación de liderazgo en costos de Porter (2000), en función de que las mejoras en el ámbito tecnológico 
Internet y televisión por cable en Venezuela: Una mirada estratégica Queipo, Beatriz; Artigas, Wileidys; Useche, María y Romero, Mildred

proporcionan apoyo para el logro de la agilización de procesos y por tanto, acortamiento de los mismos, lo cual se traduce en menores costos de producción y comercialización de los servicios que prestan. En algún momento esta estrategia puede formar parte de las estrategias de diferenciación, pero por el efecto imitador que tienen las empresas del sector, en pocos meses deja de ser una estrategia diferenciadora y pasa a ser una estrategia de liderazgo en costos. Esta automatización está determinada por lineamientos internacionales, por lo cual, se inserta dentro de las estrategias corporativas a las que hacen referencia Thompson y Strickland (2004).

e)Estrategia de modificación de la estructura organizativa.

La modificación de la estructura organizativa de las empresas que prestan el servicio de suscripción por cable e Internet se ha realizado a través de la reducción de niveles intermedios, pretendiendo generar una mayor fluidez en los procesos, en la comunicación y en la información entre los niveles organizativos. Dicha estrategia, está relacionada con la adaptación y reestructuración de las funciones organizativas a fin de hacer óptima su maniobrabilidad operativa, recurriendo a la reorientación de los recursos y esfuerzos de las distintas áreas de la empresa, para producir resultados extraordinarios; como indica Drucker (1987) integrando y unificando departamentos, unidades o gerencias en cada una de las regiones y redistribuyendo el trabajo entre los ocupantes de cada una de ellas.

En este sentido, dicha estrategia abordó dos de los elementos de la organización del trabajo; el primero, correspon- de a la especialización del trabajo horizontal, que conlleva según Chiavenato (2000) al desdoblamiento de órganos en el mismo nivel, como medio para optimizar y simplificar el cumplimiento de las tareas de los trabajadores, donde a medida que las organizaciones se desarrollaban, se subdividían en unidades paralelas relacionadas con la misma actividad o finalidad a la que se dedicaban.

El segundo elemento encontrado fue la distribución de autoridad y responsabilidad, y sus indicadores de movimientos en la cadena de mando, ya que mediante la reducción de los niveles intermedios y la redistribución del trabajo entre las demás funciones y cargos se minimizó el número de relaciones y de subordinados a supervisar.

Según la información recolectada de las empresas con esta estrategia se minimizó el número de relaciones y de subordinados a supervisar, como establece Mateo y col. (2004), contribuyendo con ello a la optimización y simplificación de las funciones organizativas y de las tareas de los trabajadores, al contar sólo con los departamentos o gerencias totalmente necesarios, lo cual hizo posible el aumento de la pericia de los trabajadores, y con ella el incremento de los niveles de eficiencia y productividad de los negocios.

Por lo tanto, con esta estrategia se modifican continuamente las estructuras organizativas en busca de la reducción de niveles, se logra el achatamiento de las mismas en búsqueda de la mejora de los procesos de retroalimentación dentro de las empresas. Reduciendo los niveles organizativos existentes, la comunicación fluye de forma más rápida y eficaz 
entre niveles; lo cual generaría una eficiencia operativa en las empresas operadoras.

Por otro lado, demostraron su capacidad de adaptarse al entorno que exigía una mayor flexibilidad en las estructuras; que sirven de enlace para los niveles del sistema, debido a que funcionó como elemento vinculante entre el medio ambiente, la organización y los subsistemas, ya que cuando se concibe la organización como un sistema abierto, se tiene un impacto sobre la estructura al establecer departamentos que controlen esta interacción o se relacionen directamente con el entorno.

Las empresas de suscripción por cable e Internet en Venezuela se han manejado a través del tiempo mediante estructuras funcionales, ocasionando la reducción o fusión de departamentos en base de una reducción de la cadena de mando para agilizar los procesos y reducir costos. Según la información recolectada, con esta estrategia se redujeron los niveles jerárquicos al eliminar un nivel de supervisión, lo cual no necesariamente significó que se disminuyó el numero de personas a supervisar o el tramo de control, porque lo que se llevo a cabo fue reasignación de las líneas de mando.

Esta modificación de la estructura en las empresas de TV por cable e Internet, consiste en la reducción de niveles organizacionales, en función del logro de la mejora en la comunicación, al mismo tiempo, que se logra la agilización de los procesos al modificar los niveles de autoridad, debido a que se reduce la burocratización de los procesos administrativos.

Los organigramas han sido modificados a lo largo de los años en función de la adaptación a los procesos implementa- dos, así como, los cambios en el entorno en donde se desarrollan las empresas de Internet y suscripción por cable al incluir nuevos servicios han modificado las estructuras y en los últimos años se han reestructurado en función de la reducción de niveles (CANTV, 2007; Intercable, 2007; Net Uno, 2007; Movistar, 2007 y Procedatos, 2007). Por otro lado, no se puede ignorar que la automatización de procesos que se ha llevado a cabo por el cambio tecnológico también ha beneficiado esta reducción de niveles.

Con estos elementos, la estrategia se inserta dentro de las de liderazgo en costos de Porter (2000), debido a que al reducir la burocracia en los procesos administrativos, se logra una reducción de los costos en función de que las decisiones son tomadas de forma más rápida, y por tanto, los servicios requeridos tienen respuesta de forma más rápida, y además, porque estructuralmente la organización se hace más simple, es decir, elimina jerarquías. En este sentido, la reducción no sólo se traduce en costos, sino también en tiempo de realización de procesos, lo cual influye en el mejoramiento de la eficiencia y efectividad del servicio. Por otro lado, esta estrategia se incluye dentro del tipo funcional de Thompson y Strickland (2004), a razón del estudio constante de los niveles organizacionales para reducir los procesos y mejorar la eficiencia.

f) Estrategia Nichos de Mercados

Para Jauregui (2007) una compañía tiene ventaja competitiva cuando cuenta con una mejor posición que los rivales para asegurar a los clientes y defenderse contra las fuerzas competitivas. Existiendo muchas fuentes de ventajas 
Internet y televisión por cable en Venezuela: Una mirada estratégica Queipo, Beatriz; Artigas, Wileidys; Useche, María y Romero, Mildred

competitivas: elaboración de productos o prestación de servicios con la más alta calidad, proporcionar un servicio superior a los clientes, lograr menores costos en los rivales, tener una mejor ubicación geográfica, diseñar un producto que tenga un mejor rendimiento que las marcas de la competencia.

La tendencia en la estrategia de las empresas es tener nichos múltiples para aumentar las oportunidades de supervivencia. Por lo que, las empresas estudiadas para ser competitivas ofrecen productos nuevos a través de diferentes planes que se adaptan a zonas residenciales, empresariales, entro otros, a precios que les permitan mantenerse en el mercado.

Algunas de las empresas estudiadas (CANTV, 2007 y Procedatos, 2007) poseen productos y/o servicios adaptados a las nuevas tendencias de segmentos de mercado, como en el caso específico del surgimiento en los últimos años de PYMES y Cooperativas, para lo cual se ofrecen productos y servicios según las necesidades de las mismas, con tarifas ajustadas y productos según los requerimientos. Además por la ley de responsabilidad social, las empresas han tenido que penetrar en otros nichos de mercado y ofrecer servicios adaptados a las realidades de los mismos.

Esta estrategia está relacionada con el desarrollo de productos y/o servicios en función del logro de cierta cuota de mercado, como el caso de productos específicos para los nuevos sectores, como el caso de las PYMES, cooperativas, empresas de cogestión, entre otros. En el caso específico de CANTV (2007) desarrolla productos para nichos específicos, sin dejar de lado, que con la nacio- nalización se espera llegar a nichos de mercado que han sido excluidos de los servicios que se prestan, garantizando así la reducción de la exclusión tecnológica de la cual son objeto.

Esta estrategia se inserta dentro de las estrategias de diferenciación de Porter (2000), en función de lograr que el cliente diferencie la empresa en el mercado por los productos y/o servicios que ofrece. Así mismo, se inserta dentro de las estrategias de negocio de Thompson y Strickland (2004), debido que al orientar el negocio no sólo a las mayorías o a los que pueden pagar el servicio, se logra modificar su visión del mercado y establecer lineamientos específicos que regirán las organizaciones como negocios específicos en el ámbito nacional.

Se puede decir, que las estrategias se insertan en su mayoría como estrategias de liderazgo en costos, así como, el nivel preponderante es el nivel corporativo. En primera instancia, las empresas logran establecer la mayoría de sus estrategias en función de diferenciarse por costos bajos, ofreciendo así un mejor precio en el mercado, y el otro tipo de estrategias lo establecen en función de poder cumplir con los mandatos de la casa matriz o de las corporaciones internacionales como parte de las mismas, muy poco se observa la particularización de los productos y/o servicios y el uso de otras estrategias de carácter local.

\section{Conclusiones}

Las empresas de Internet y Televisión por cable en Venezuela deben persistentemente optimizar la eficacia de sus actividades y para ello deben contar con una perspectiva estratégica que les per- 
mita la búsqueda constante del mejoramiento de sus sistemas, procesos, procedimientos, productos y/o servicios con la finalidad de adaptarse a un mercado interno, caracterizado por la apertura, liberación de las telecomunicaciones, por ello han desarrollado estrategias en la organización del trabajo que consigan ventaja competitiva.

Las empresas abordadas implementaron estrategias en la organización del trabajo con elementos organizativos destacados representada en la división del trabajo, la especialización, rotación del personal, la automatización de los procesos y creación de nichos de mercados; realizando cambios en las estructuras de las empresas, también manejaron elementos de los centrados en los avances en materia organizacional, de la administración contemporánea y actual.

En consecuencia, pudiéramos estar en presencia de la conformación de unas estrategias adaptadas a las nuevas exigencias del mundo globalizante, donde el avance de la ciencia y la tecnología obligan a las empresas abordar de una manera nueva la realidad organizacional, por lo tanto, es importante señalar que la presencia del hombre como ente pensante y transformador de su realidad busca por todos los medios posible del conocimiento alcanzar cada día su objeto básico, como es obtener el dominio total del mercado.

Por otro lado, en cuanto a la orientación estratégica, se pudo constatar en las empresas de internet y televisión por cable que las estrategias implementadas en su mayoría responden a la búsqueda del liderazgo en costos, a fin de optimizar el uso de los recursos y el logro de una mayor rentabilidad, que a su vez permita el posicionamiento en el mercado. En cuanto a las estrategias de diferenciación, las mismas son temporales, en función de la rápida imitación que existe entre las empresas del sector.

Por último, cuando se hace referencia al nivel en el cual se desarrollan las estrategias, las mismas en su mayoría son de nivel corporativo, en función del cumplimiento de los lineamientos establecidos por las grandes corporaciones a las cuales se encuentran subordinadas dichas organizaciones, así como en el caso de las empresas nacionales, rigiéndose por los lineamientos de la casa matriz; sin dejar de lado el establecimiento también de estrategias de negocios y funcionales.

Si se establecen elementos diferenciadores en dichas estrategias, no se pueden observar certeramente, debido a que como se señala en el caso de las estrategias de diferenciación, la capacidad imitativa es bastante alta, en función del uso de recursos para incrementar sus herramientas tecnológicas, así como, sus procesos operacionales, sin embargo, no se puede dejar de lado, que se observa mayor desarrollo tecnológico en aquellas empresas mejor posicionadas en el mercado, así como, mayor inclinación al mejoramiento en operaciones para aquellas que no cuentan con una capacidad de inversión elevada. 
Internet y televisión por cable en Venezuela: Una mirada estratégica

Queipo, Beatriz; Artigas, Wileidys; Useche, María y Romero, Mildred

\section{Referencias Bibliográficas}

Brickley, J.; Smith, C y Zimmerman, L. (2005). Diseño de organizaciones para crear valor. Editorial Graw Hill. Colombia.

Chiavenato, Idalberto (2000). Introducción a la Teoría General de la Administración Editorial Mc Graw Hill. Colombia.

Chiavenato, Idalberto (2006). Introducción a la Teoría General de la Administración. Editorial MC Graw Hill. México.

Corporación Andina de Fomento (1991). Gestión Financiera. Editorial Nuevos tiempos. Venezuela.

Drucker, Peter (1987). La Gerencia. Tareas, Responsabilidades y practicas (6ta edición). Librería el Ateneo Editorial. Argentina.

Escorsa, Castells y Valls, Juane (2003). Tecnología e innovación en la Empresa. Ediciones Politetext. España.

Fernández, Esteban; Avella, Lucía y Fernández, Martha (2003). Estrategia de Producción. Editorial Graw Hill. España.

Gual, Jordi (2001). Estrategias empresariales en Infocom: El nuevo sector de telecomunicaciones e internet. Revista Nuevas Tecnologias ¿Nueva economia?, agosto-septiembre 2001, numero 793. Pag. 77-87.

Jauregui, Ana (2007). Estrategia y ventaja competitiva. Extraído de www.monografias.com/trabajos13/esven/esven.shtml en noviembre de 2007.

Koontz, Harold y Weihrich, Heinz. (1994). Administración. Una perspectiva global. Editorial Mc Graw Hill. Colombia.

Kotler, Peter; Armstrong, Gary; Cámara, Dionisio y Cruz, Ignacio (2004). Marketing (10ma edición). Editorial Pearson. España.
Molina, Hipolito y Conca, Francisco (2000). Innovación tecnológica y Competitividad Empresarial. Publicaciones. Universidad de Alicante. Argentina.

Mateo, Carlos; Peyrú, Pablo; Verna y Etchebe, Roberto (2004). La escuela neoclásica. Ingeniería industrial II. Documento en línea. Extraído de www.monografias.com/roberto_verna@hotmail.com, en fecha octubre 2007.

Melinkoff, Ramón (1990). La estructura de la organización. Los organigramas. Editorial Panapo. Venezuela.

Porter, Michael (2000). Estrategia Competitiva. Técnicas para el analisis de los sectores industriales y la competencia. Cecsa, Mexico.

Porter, Michael (2002). Ventaja competitiva (edición revisada). Compañía editorial continental. México.

Thompson, Arthur y Strickland, A.J. (2004). Administración Estratégica. Textos y Casos. Mc Graw Hill, México.

Zeithaml, Valerie y Bitner, Mary (2000). Marketing de los servicios. Un enfoque de integración del cliente a la empresa. Editorial Mc Graw Hill. México.

\section{Entrevistas Realizadas}

CANTV (2007). Entrevista realizada en el área administrativa.

Intercable (2007). Entrevista realizada en las áreas de administración y operaciones.

Movistar (2007). Entrevista realizada en el area de administración.

Net Uno (2007). Entrevista realizada en las áreas de mercadeo y operaciones.

Procedatos (2007). Entrevista realizada en las áreas de administración y operaciones. 